\title{
Novel Metallic Clathrates of Group-IV Elements and Their Compounds in a Dense Hexagonal Lattice
}

\author{
Taylan Gorkan, ${ }^{\dagger}$ Ilkay Ozdemir, ${ }^{\dagger}$ M. Yagiz Bakir, ${ }^{\dagger}$ Fatih Ersan, ${ }^{\dagger} \odot$ Gökhan Gökoğlu, ${ }^{\dagger}$ \\ Ethem Aktürk, ${ }^{*},, \mathbb{A} \odot$ and Salim Ciraci*, ${ }^{*}$ \\ ${ }^{\dagger}$ Department of Physics and "IN Nanotechnology Application and Research Center, Adnan Menderes University, 09100 Aydın, Turkey \\ ${ }^{\ddagger}$ Department of Mechatronics Engineering, Faculty of Engineering, Karabuk University, 78050 Karabuk, Turkey \\ ${ }^{\S}$ Department of Physics, Bilkent University, 06800 Ankara, Turkey
}

\section{Supporting Information}

\begin{abstract}
Further, to recently introduced metallic $\mathrm{NaSi}_{6}$ and $\mathrm{Si}_{6}$ clathrate structures, we show that not only $\mathrm{Si}$ but also other group-IV elements, such as $\mathrm{C}, \mathrm{Ge}$, and $\mathrm{Sn}$, can form stable and metallic clathrate structures with open channels at the corners of hexagons. These elemental clathrates of $\mathrm{Si}, \mathrm{Ge}$, and $\mathrm{Sn}$ can be viewed as if they are a combination of 2D metallic planes and perpendicular 1D metallic chains, the interplay of which can give rise to interesting physical effects. When free-standing, these atomic planes transform to $2 \mathrm{D}$ semiconducting, single-layer structures. The clathrate structure of $\mathrm{C}$, which consists of weakly interacting, vertical hexagonal tubes situated at the corners of a $2 \mathrm{D}$ hexagonal lattice, is insulating in the plane but $1 \mathrm{D}$ metallic perpendicular to the planes. We also show that stable compound clathrate structures can form by hosting different alkali, alkaline earth, and light transition metal atoms in the open channels of elemental clathrates. These new metallic allotropes of group-IV elements predicted by first-principles calculations based on the density functional theory exhibit features that can be critical fundamentally and technologically.
\end{abstract}

\section{INTRODUCTION}

Silicon ( $\mathrm{Si})$ is the most important, insofar as one of the most abundant group-IV elements on Earth. The cubic diamond (CD) structure, namely, the phase corresponding to the global minimum, has an open structure held by tetrahedrally coordinated covalent bonds constructed by $\mathrm{sp}^{3}$-bond orbitals and high metallicity according to the scale of the bond-orbital model. ${ }^{1-3}$ The fundamental band gap of $1.1 \mathrm{eV}$, excellent mechanical properties, and capacity to be grown into a largesize single crystal have made silicon in the $C D$ structure the most crucial ingredient of modern microelectronics. The vision of using well-developed silicon technology in flexible 2D nanoelectronics has led to the prediction of stable Si singlelayers, namely, silicene, ${ }^{4,5}$ which was already grown on a $\mathrm{Ag}(111)$ substrate in single-layer and multilayer forms. Threedimensional (3D) layered stable, eclipsed, and staggered structures of Si constructed by stacking trigonal and hexagonal dumbbell single-layers ${ }^{6}$ named as silicite ${ }^{7}$ appear to be a challenge at the experimental site. Besides, silicon can also be grown in the wurtzite structure and in various metastable forms such as amorphous, porous clathrates.

Semiconducting silicon in the CD structure can undergo a metal-insulator transition at high pressure, in extreme conditions realized in a diamond anvil cell. The transition follows the sequence $\mathrm{Si}-1 \rightarrow \mathrm{Si}-2(\beta$-Sn structure) at about 10 $\mathrm{GPa} \rightarrow \mathrm{Si}-11($ Imma-Si) $\rightarrow \mathrm{Si}-5(\mathrm{SH}$, simple hexagonal structure) at about $15 \mathrm{GPa} \rightarrow \mathrm{Si}-6(\mathrm{Cmca}-\mathrm{Si})$ at about $38 \mathrm{GPa}$ $\rightarrow \mathrm{Si}-7$ (hexagonal close-packed) at about $40 \mathrm{GPa} \rightarrow \mathrm{Si}-10$ (face-centered cubic) at about $80 \mathrm{GPa}^{8-14}$

Germanium, being another group-IV element just below $\mathrm{Si}$, shows also a phase transition from a semiconducting CD structure to a $\beta$-Sn structure at $10 \mathrm{GPa}$, to Imma at $75 \mathrm{GPa}$, to $\mathrm{SH}$ at $85 \mathrm{GPa}$, to $\mathrm{Cmca}$ at $100 \mathrm{GPa}$, and to hcp at $170 \mathrm{GPa}{ }^{15}$ Although the sequence of the structural transitions of $\mathrm{Ge}$ is similar to $\mathrm{Si}$, transition pressures are higher than $\mathrm{Si}$ due to $3 \mathrm{~d}$ core states behind the $4 \mathrm{~s}$ and $4 \mathrm{p}$ valence states.

However, due to strong kinetic effects, the structural transitions occurring at a high pressure are not completely reversible upon pressure release at ambient temperature. This causes various metastable phases to form through pressure release such as $\mathrm{Si}-12$ (R8, rhombohedral) and $\mathrm{Si}-3$ (BC8, body-centered). ${ }^{13,16-18}$ Subsequent thermal annealing of BC8$\mathrm{Si}$ results in Si-4 (hexagonal diamond) formation. ${ }^{13,17}$ By rapid release of pressure from the metallic state, two metastable phases of $\mathrm{Si}$ with a tetragonal structure, referred as $\mathrm{Si}-8$ and $\mathrm{Si}$ 9, have been discovered. ${ }^{19}$ Other metastable phases with a tetragonal structure of Si, BT8 and ST12, have been produced by Rapp et al. by microexplosion experiments and computa-

Received: April 8, 2019

Revised: May 10, 2019

Published: May 22, 2019 
tional research within ab initio calculations. ${ }^{20}$ They have reported that these new phases are likely to exhibit interesting properties, such as predicted narrow band gap semiconductor (BT8-Si) and potentially superconducting behavior (ST12-Si).

Numerous studies on the synthesis of other Si allotropes by using appropriate chemical precursors followed by physical and chemical manipulations have been published. $9,10,13,17,19-27$ However, up until the study of Sung et al., ${ }^{28}$ the reported metastable Si allotropes were semiconducting; pure metallic Si phases at ambient conditions have not been investigated yet. Sung et al. ${ }^{28}$ reported the prediction of pure metallic Si allotropes with open channels at ambient pressure and named it $\mathrm{P} 6 / \mathrm{m}-\mathrm{Si}_{6}$. They show that this metallic phase can be obtained by removing $\mathrm{Na}$ after pressure release from a novel $\mathrm{Na}-\mathrm{Si}$ clathrate called $\mathrm{P} 6 / \mathrm{m}-\mathrm{NaSi}_{6}$. To examine low-enthalpy $\mathrm{Na}-\mathrm{Si}$ allotropes with various composition ratios, they utilized an ab initio evolutionary crystal structure search method that combines conformational space annealing algorithm ${ }^{29}$ for global optimization and first-principles electronic structure calculations. Furthermore, they have confirmed that both P6/ $\mathrm{m}-\mathrm{NaSi}_{6}$ and $\mathrm{P} 6 / \mathrm{m}-\mathrm{Si}_{6}$ are stable and can be superconductors with the critical temperatures of about 13 and $12 \mathrm{~K}$ at zero pressure, respectively. It is estimated that all of these metastable $\mathrm{Si}$ allotropes exhibit a wide range of features not only scientifically but also technologically. Moreover, the success in the exploration of new Si phases raises the question of whether other similar materials can form.

In this study, we investigated clathrates of group-IV elements, namely, $\mathrm{P} 6 / \mathrm{m}-\mathrm{A}_{6}$ (or shortly $\mathrm{A}_{6}$ ) and $\mathrm{P} 6 / \mathrm{m}-\mathrm{XA}_{6}$ compounds (or $\mathrm{XA}_{6}$ ) (with $\mathrm{A}=\mathrm{C}, \mathrm{Si}, \mathrm{Ge}$, and $\mathrm{Sn}$; alkali metal atoms $\mathrm{X}=\mathrm{Li}, \mathrm{Na}, \mathrm{K}$; alkaline earth metal atoms $\mathrm{X}=\mathrm{Mg}, \mathrm{Ca}$, $\mathrm{Be}$; $3 \mathrm{~d}$ transition metal atoms $\mathrm{X}=\mathrm{Sc}, \mathrm{Ti}$ ), based on firstprinciples density functional theory (DFT). Revealing clathrates beyond $\mathrm{Si}$ and $\mathrm{Na}-\mathrm{Si}$, increasing their density of states at the Fermi level, $D\left(E_{\mathrm{F}}\right)$, with divalent atoms, and attaining magnetic properties with transition metal atoms have been the prime motivations for considering so many atoms. The stability of all clathrates, $\mathrm{P} 6 / \mathrm{m}-\mathrm{A}_{6}$ and $\mathrm{P} 6 / \mathrm{m}-\mathrm{XA}_{6}$, have been examined first based on dynamical and thermal stability analyses. Then, the structural parameters and cohesive energy of the stable structures have been calculated; their energy band structures and the character of bonds between the two nearest group-IV atoms and the metallic binding have been analyzed. With the hope of monitoring the density of states at the Fermi level and, hence, the transition temperature from normal to superconducting state $T_{\mathcal{c}}$, the response of the $\mathrm{P} 6 / \mathrm{m}-\mathrm{Si}_{6}$ structure to the applied biaxial compressive and tensile strain has been examined. Finally, the stability of a two-dimensional (2D) single-layer to be cleaved from the $\mathrm{P} 6 / \mathrm{m}-\mathrm{Si}_{6}$ crystal, which has an atomic structure similar to $\omega$-borophene, ${ }^{30}$ has been investigated. The important and original findings of our paper are summarized as follows: (i) P6/m-A 6 ( $=\mathrm{C}, \mathrm{Ge}, \mathrm{Sn})$ structures are dynamically and thermally stable at low and elevated temperatures. All of these structures are metallic with remarkable electronic density of states at $E_{\mathrm{F}}$. (ii) The optimized $\mathrm{P} 6 / \mathrm{m}-\mathrm{C}_{6}$ (or $\mathrm{C}_{6}$ ) structure is composed of tubes of hexagons, which are brought together by weak van der Waals interaction. The system behaves as conducting hexagonal rods perpendicular to the $x y$ plane, indicating onedimensional (1D) conduction. The wide electronic barriers between hexagonal tubes hinder conduction in the $x y$ plane. (iii) $\mathrm{A}_{6}(\mathrm{~A}=\mathrm{Si}, \mathrm{Ge}, \mathrm{Sn})$ clathrates also form $1 \mathrm{D}$ metallic chains along the $z$-axes. However, $\mathrm{Si}_{6}, \mathrm{Ge}_{6}$, and $\mathrm{Sn}_{6}$ permit $1 \mathrm{D}-2 \mathrm{D}$ conduction mixed in a 3D lattice. (iv) Both tensile and compressive strains in $\mathrm{Si}_{6}$ decrease DOS at $E_{\mathrm{F}}$, yielding lower electronic conduction. ( $\mathrm{v}$ ) We found that alkali, alkaline earth, and transition metals including $16 \mathrm{P} 6 / \mathrm{m}^{-\mathrm{XA}_{6}}$ clathrate systems are both dynamically and thermally stable. These systems also depict metallic behavior.

\section{COMPUTATIONAL DETAILS}

We have performed first-principles calculations for total energy and electronic structure within DFT by using the plane-wave basis set and projector augmented-wave (PAW) potentials ${ }^{31}$ as implemented in the Vienna $a b$ initio simulation package (VASP). ${ }^{32,33}$ The exchange-correlation potential is approximated with generalized gradient approximation (GGA) using Perdew-Burke-Ernzerhof (PBE) exchange correlation functional $^{34}$ including van der Waals (vdW) correction. ${ }^{35}$ We have considered $2 s^{1} 2 p^{0}, 3 s^{1} 3 p^{0}$, and $3 s^{1} 3 p^{6} 4 s^{1}$ configurations for $\mathrm{Li}$, $\mathrm{Na}$, and $\mathrm{K}$, respectively; $2 \mathrm{~s}^{2} 2 \mathrm{p}^{0}, 3 \mathrm{~s}^{2} 3 \mathrm{p}^{0}$, and $3 \mathrm{~s}^{2} 3 \mathrm{p}^{6} 4 \mathrm{~s}^{2}$ for $\mathrm{Be}$, $\mathrm{Mg}$, and Ca, respectively; $2 \mathrm{~s}^{2} 2 \mathrm{p}^{2}, 3 \mathrm{~s}^{2} 3 \mathrm{p}^{2}, 4 \mathrm{~s}^{2} 4 \mathrm{p}^{2}$, and $5 \mathrm{~s}^{2} 5 \mathrm{p}^{2}$ for C, Si, Ge, and Sn, respectively; $3 \mathrm{p}^{6} 4 \mathrm{~s}^{2} 3 \mathrm{~d}^{1}$ for Sc; and $4 \mathrm{~s}^{2} 3 \mathrm{~d}^{2}$ for $\mathrm{Ti}$ as valence electrons. By using the conjugate-gradient algorithm, ${ }^{36,37}$ all the atomic positions and lattice parameters are fully optimized until the forces on each atom are less than $1.0 \times 10^{-4} \mathrm{eV} / \AA$. The convergence criterion of self-consistent calculations is taken to be $10^{-5} \mathrm{eV}$ for total energy values. Pressures on the lattice unit cell are reduced to values less than $0.5 \mathrm{kB}$. The partial occupancies are determined using the Methfessel-Paxton scheme ${ }^{38}$ with the smearing width of 0.2 $\mathrm{eV}$. For the Brillouin zone (BZ) integration in the $\mathbf{k}$-space, a set of $(15 \times 15 \times 30)$ k-point sampling is used within the Monkhorst-Pack scheme. ${ }^{39}$ For the charge-transfer analysis, the effective charge on atoms is obtained using the Bader method. ${ }^{40}$ The cohesive energy of an elemental clathrate (per atom), $E_{\mathrm{c}}\left[\mathrm{A}_{6}\right]$, is calculated using the expression $E_{\mathrm{c}}\left[\mathrm{A}_{6}\right]=$ $\left(6 E_{\mathrm{T}}[\mathrm{A}]-E_{\mathrm{T}}\left[\mathrm{A}_{6}\right]\right) / n$, with $n=6$. Similarly, the average cohesive energy of a compound clathrate is $E_{\mathrm{c}}\left[\mathrm{XA}_{6}\right]=\left(E_{\mathrm{T}}[\mathrm{X}]\right.$ $\left.+6 E_{\mathrm{T}}[\mathrm{A}]-E_{\mathrm{T}}\left[\mathrm{XA}_{6}\right]\right) / n$. Here, $E_{\mathrm{T}}\left[\mathrm{A}_{6}\right], E_{\mathrm{T}}\left[\mathrm{XA}_{6}\right], E_{\mathrm{T}}[\mathrm{A}]$, and $E_{\mathrm{T}}[\mathrm{X}]$, respectively, are the total energies of the elemental clathrate, compound clathrate, free $\mathrm{A}$ atom, and free $\mathrm{X}$ atom. $n$ is the number of atoms in a unit cell. The formation energy (per atom), $E_{f}$, of an elemental clathrate $A_{6}$ is obtained by subtracting the cohesive energy of its counterpart in the $C D$ structure corresponding to a global minimum from, that is, $E_{\mathrm{f}}=$ $E_{\mathrm{c}}\left[\mathrm{A}_{6}\right]-E_{\mathrm{c}}[\mathrm{CD}-\mathrm{A}]$.

Phonon dispersion spectra are computed using force constants of the supercell within the framework of densityfunctional perturbation theory (DFPT) ${ }^{41}$ as implemented in the VASP code combined with PHONOPY package. ${ }^{42}$ In phonon calculations, an improved energy convergence criterion as $10^{-8} \mathrm{eV}$ is attained to obtain well-converged realspace force constants. These calculations were performed using $2 \times 2 \times 2$ supercells. We have also carried out the thermal stability analysis of the systems by performing ab initio molecular dynamics (AIMD) simulations at 300, 500, and $1000 \mathrm{~K}$. All the structures are visualized using the VESTA code. $^{43}$

\section{METALLIC 3D P6/M-A 6 ALLOTROPES OF GROUP-IV ELEMENTS}

The $\mathrm{P} 6 / \mathrm{m}$ clathrate structure has a simple $3 \mathrm{D}$ hexagonal lattice, allowing open channels to host suitable $\mathrm{X}$ metal atoms between adjacent hexagonal rings in the $x y$-plane. Group-IV 


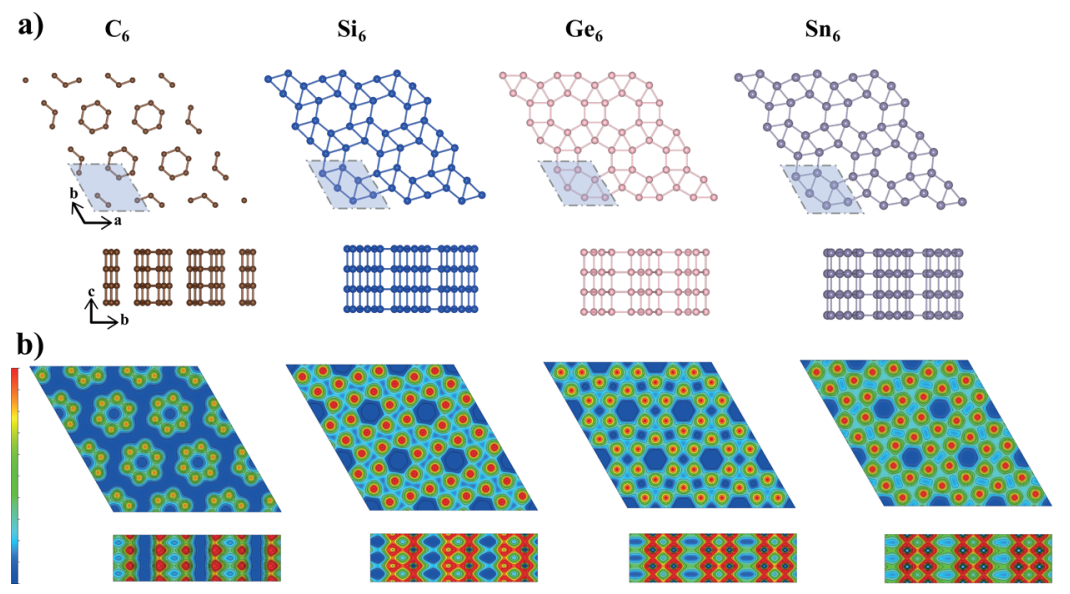

Figure 1. (a) Top ( $x y$ plane) and side views of the optimized atomic structure of the clathrate structures of group-IV elements $P 6 / \mathrm{m}^{-\mathrm{A}_{6}}$ (or simply $\mathrm{A}_{6}$ ) with $\mathrm{A}=\mathrm{C}, \mathrm{Si}, \mathrm{Ge}$, and $\mathrm{Sn}$. Hexagonal primitive unit cells are shaded. (b) Contour plots of the total charge density at the lateral $x y$-plane passing through the hexagons and at the vertical plane. The color scale at the left side indicates shift from high density (red) to low density (dark blue). Open channels are shown as dark blue spots, and $1 \mathrm{D}$ conducting chains of atoms are shown as red/orange ribbons.

atoms, that is, A atoms, have five neighbors in the $x y$-plane and two neighbors in the adjacent atomic planes just below and above. This system is composed of 7-fold coordinated A atoms connected to seven neighboring $\mathrm{A}$ atoms with $\mathrm{A}-\mathrm{A}$ bonds with slightly differing bond lengths. On the other hand, these A-A bonds are longer than the counterparts in the CD structure. In a recent work of Sung et al., ${ }^{28}$ it was reported that the $\mathrm{P} 6 / \mathrm{m}$ $\mathrm{NaSi}_{6}$ clathrate structure can be synthesized at high pressure $(12.4 \mathrm{GPa})$ conditions and the stability is preserved down to ambient pressure. Moreover, the pure $\mathrm{P} 6 / \mathrm{m}-\mathrm{Si}_{6}$ system can be obtained by removing $\mathrm{Na}$ atoms from channels via thermal degassing. In the literature, this was the first metallic Si system that can be stabilized at ambient pressure. In the present work, we examine the clathrates of group-IV elements $(A=C, G e$, $\mathrm{Sn}$ ) in the $\mathrm{P} 6 / \mathrm{m}$ structure. We included $\mathrm{Si}$ for the sake of comparison. The structures of the elemental (or bare) clathrates $\mathrm{A}_{6}(\mathrm{~A}=\mathrm{C}, \mathrm{Si}, \mathrm{Ge}$, and $\mathrm{Sn})$ are described in Figure 1a.

Structure Optimization and Stability. The calculated structural parameters as well as cohesive and formation energies of the elemental clathrates are presented in Table 1, together with those calculated for the counterparts in the CD structure. Our results related with the compound clathrates $\mathrm{XA}_{6}$ will be presented in the forthcoming sections.

All $\mathrm{A}_{6}$ crystals have positive cohesive energy, indicating that they are favorable energetically relative to their free atom constituents. This however does not ensure that these structures are structurally stable. Later, we will demonstrate their stability by the dynamical and thermal stability analyses. Normally, the cohesive energy of a clathrate $A_{6}$ is smaller than that of the counterpart in the $\mathrm{CD}$ structure in the global minimum. The difference is high in $\mathrm{C}_{6}$ clathrate. Consistent with this conclusion, the calculated formation energies of $\mathrm{C}_{6}$, $\mathrm{Si}_{6}$, and $\mathrm{Ge}_{6}$ are negative. Notably, $E_{\mathrm{f}}$ of $\mathrm{Sn}_{6}$ is slightly positive. As for the A-A distances, they are significantly larger than the covalent bond lengths of the counterparts in the $\mathrm{CD}$ structure. For example, the $\mathrm{Si}-\mathrm{Si}$ distances of $\mathrm{Si}_{6}$ ranging between 2.48 and $2.62 \AA$ are larger than the covalent bond of $\mathrm{Si}$ in the CD structure calculated as $d=2.34 \AA$.

Even if one starts from an initial clathrate structure $\mathrm{P} 6 / \mathrm{m}-\mathrm{C}_{6}$ for $\mathrm{C}$ such as $\mathrm{Si}_{6}$ clathrate, the structure has changed to the clathrate structure shown in Figure 1a upon optimization. This
Table 1. Values Calculated by Using PBE for P6/m-A $(A=$ $\mathrm{C}, \mathrm{Si}, \mathrm{Ge}$, and $\mathrm{Sn}$ ) and Their Counterparts in Cubic Diamond (CD) Structures ${ }^{a}$

\begin{tabular}{|c|c|c|c|c|c|}
\hline \multirow[b]{2}{*}{ structure } & \multicolumn{2}{|c|}{$\begin{array}{l}\text { lattice } \\
\text { constants }\end{array}$} & \multirow[b]{2}{*}{$\begin{array}{c}\min . \\
\text { distance } \\
d(\AA)\end{array}$} & \multirow[b]{2}{*}{$\begin{array}{c}\text { cohesive energy } \\
E_{\mathrm{c}}(\mathrm{eV} / \text { atom })\end{array}$} & \multirow{2}{*}{$\begin{array}{l}\text { formation } \\
\text { energy } E_{\mathrm{f}} \\
(\mathrm{eV} / \text { atom })\end{array}$} \\
\hline & $a(\AA)$ & $c(\AA)$ & & & \\
\hline $\mathrm{C}_{6}$ & 5.80 & 1.61 & 2.25 & 6.03 & -1.91 \\
\hline $\mathrm{Si}_{6}$ & 6.74 & 2.49 & 2.48 & 4.50 & -0.33 \\
\hline $\mathrm{Ge}_{6}$ & 7.27 & 2.65 & 2.62 & 3.85 & -0.21 \\
\hline $\mathrm{Sn}_{6}$ & 8.25 & 2.99 & 2.99 & 3.37 & 0.02 \\
\hline $\mathrm{CD}-\mathrm{C}$ & 3.56 & & 1.54 & 7.94 & \\
\hline $\mathrm{CD}-\mathrm{Si}$ & 5.41 & & 2.34 & 4.83 & \\
\hline $\mathrm{CD}-\mathrm{Ge}$ & 5.66 & & 2.45 & 4.06 & \\
\hline $\mathrm{CD}-\mathrm{Sn}$ & 6.46 & & 2.81 & 3.35 & \\
\hline
\end{tabular}

${ }^{a}$ Lattice constants, $|\mathbf{a}|=|\mathbf{b}|=a$ and $|\mathbf{c}|=c$; minimum A-A distance, $d$; cohesive energy, $E_{\mathrm{c}}$; formation energy, $E_{\mathrm{f}}$.

structure is constructed from the tubes of hexagons, the centers of which are placed at the corners of the $2 \mathrm{D}$ hexagonal lattice. Nonetheless, it has a hexagonal lattice with a primitive unit cell containing six $\mathrm{C}$ atoms. The $\mathrm{C}-\mathrm{C}$ distance between the atomic planes, namely, $c$, is $40 \%$ shorter than the in-plane $\mathrm{C}-\mathrm{C}$ bonds. In this respect, the $\mathrm{C}_{6}$ structure does not depict a layered character. On the other hand, the $\mathrm{C}-\mathrm{C}$ bonds constructing hexagonal rings in the $x y$ plane form strong bonds with a length comparable to that of graphene. Therefore, in the top view, $\mathrm{C}_{6}$ appears as if it is graphene made of disconnected hexagons. The charge density isosurfaces depict the tubular character, with a very low charge density inside and around hexagonal tubes. Accordingly, it is expected that the hexagonal tubes are brought together by weak van der Waals interaction to form a $\mathrm{C}_{6}$ structure. Such a situation occurs in $3 \mathrm{D}$ trigonal selenium and tellurium, where weakly interacting chiral tubes along the $z$ direction are located also at the corners of a $2 \mathrm{D}$ hexagonal lattice.

Starting first from $\mathrm{P} 6 / \mathrm{m}-\mathrm{Si}_{6}$, the lattice constants calculated in the present study, $a=6.74 \AA$ and $c=2.49 \AA$, are in agreement with those calculated in ref 28 . The cohesive energy of $\mathrm{Si}_{6}$ is calculated as $E_{\mathrm{c}}=4.50 \mathrm{eV}$ per atom, comparable to that of the $\mathrm{CD}$ counterpart, which is calculated as $4.83 \mathrm{eV}$ 

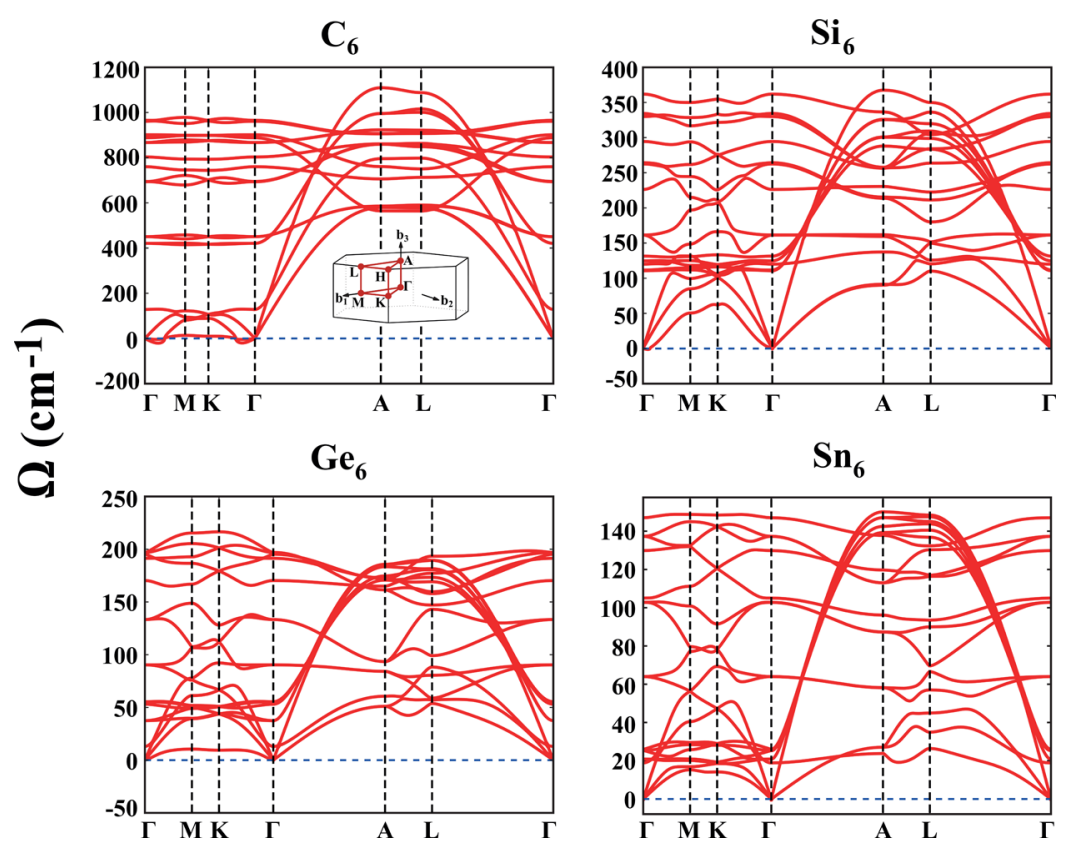

Figure 2. Phonon dispersion curves, that is, $\Omega(\mathbf{k})$, along major symmetry directions of the Brillouin zone calculated for the optimized structures of $\mathrm{C}_{6}, \mathrm{Si}_{6}, \mathrm{Ge}_{6}$, and $\mathrm{Sn}_{6}$. The Brillouin zone is described in the inset.
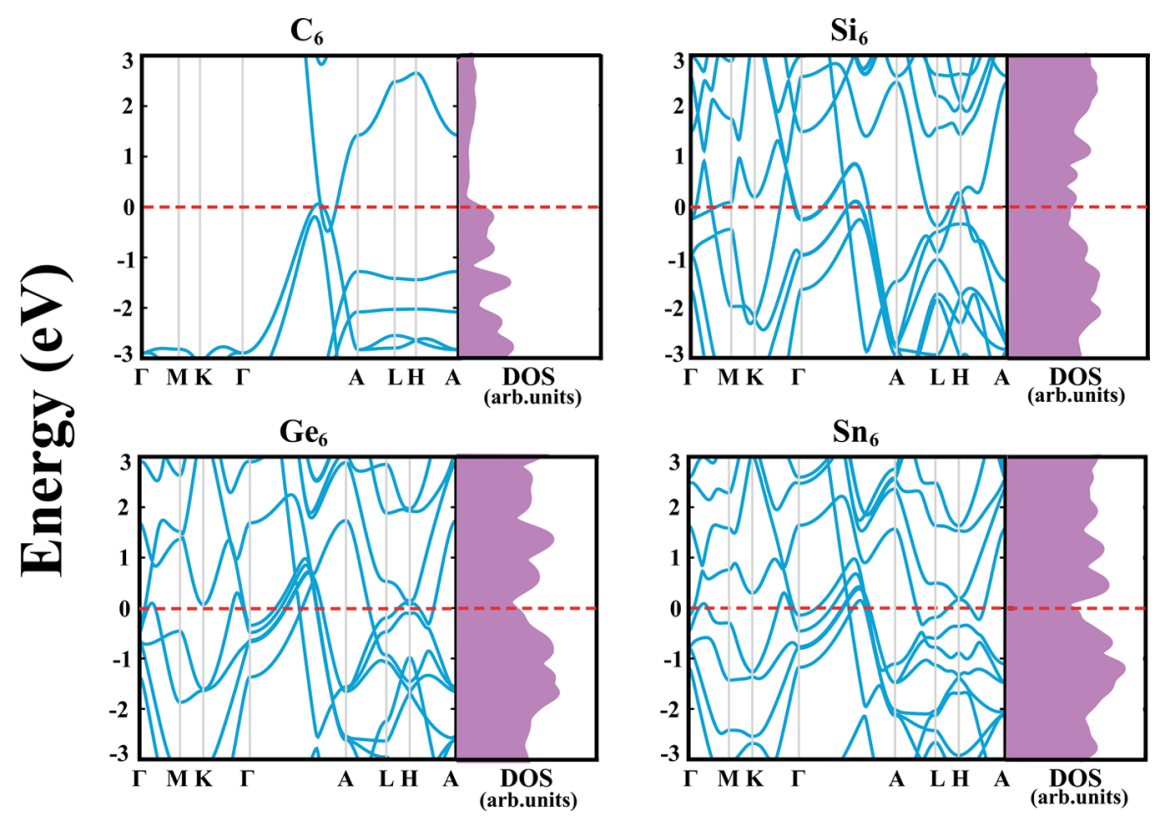

Figure 3. Electronic energy band structure $E_{n}(\mathbf{k})$ along major symmetry directions of the Brillouin zone and corresponding density of states (DOS) calculated for $\mathrm{C}_{6}, \mathrm{Si}_{6}, \mathrm{Ge}_{6}$, and $\mathrm{Sn}_{6}$. The zero of energy is set at the Fermi level and is shown by dashed lines.

(4.63 eV/atom determined experimentally). Table 1 and Figure la show that $\mathrm{Ge}_{6}$ and $\mathrm{Sn}_{6}$ clathrate structures display trends that are similar to those of $\mathrm{Si}_{6}$. Charge density contour plots indicate that $\mathrm{Si}_{6}, \mathrm{Ge}_{6}$, and $\mathrm{Sn}_{6}$ clathrates are rather different from $\mathrm{C}_{6}$ : all group-IV clathrates form metallic $1 \mathrm{D}$ vertical chains along the $z$ axis, whereas the electronic charges of $\mathrm{Si}_{6}, \mathrm{Ge}_{6}$, and $\mathrm{Sn}_{6}$ are delocalized in the $x y$ atomic planes as a result of 5-fold coordination and constitute metallic bonding. Conversely, the charge of $\mathrm{C}_{6}$ in the $x y$ atomic planes of hexagons is localized between the $\mathrm{C}-\mathrm{C}$ bonds of the hexagons. The implications of this distribution will be discussed in the forthcoming sections.
The dynamical stability of the elemental clathrates $A_{6}$ has been tested by the calculation of the frequencies of vibration modes. The eigenvalues of dynamical matrix, namely, the square of specific frequencies, $\Omega(\mathbf{k})^{2}$, would be negative in the absence of sufficient restoring force and hence could not return to its initial equilibrium position. At the end, the initial structure would be transformed to a different structure. Here, the calculated phonon dispersion curves of all $\mathrm{A}_{6}$ clathrate structures presented in Figure 2 have positive frequencies along the symmetry axis of BZ. Only near the center of BZ, namely, $\lambda \rightarrow \infty$, specific modes can get imaginary values, which is well known to be an artifact of the numerical calculations. Notably, the phonon dispersion curves calculated 
for $\mathrm{Si}_{6}$ are in agreement with those calculated by Sung et al. ${ }^{28}$ The dimensionality is well recognized in the phonon dispersions: While in-plane atoms have significant dispersion for the $\mathbf{k} \| x y$-plane, their dispersion is not high along the $z$ axis. On the other hand, the optical modes, which are rather flat dispersion along $\Gamma-\mathrm{M}-\mathrm{K}-\Gamma$ directions, attain high dispersion along the $\Gamma-\mathrm{A}$ direction, which is related with the metallic chain atoms along the $z$ direction. These atoms display also a longitudinal branch with high dispersion along the $\Gamma-\mathrm{A}$ direction.

We also performed crude thermal stability analysis, which verifies that the elemental clathrate structures resist to thermal excitations at a high temperature. Based on this stability analysis, we concluded that the clathrate structures of group-IV elements keep their structural stability above the room temperature.

Electronic Energy Structure. The electronic band structures of the optimized $\mathrm{A}_{6}$ clathrate structures and the densities of states are presented in Figure 3. The clathrate structures of four elements are metallic with several bands crossing the Fermi level. Because of the atomic configuration of $\mathrm{C}_{6}$, which is rather different from $\mathrm{Si}_{6}, \mathrm{Ge}_{6}$, and $\mathrm{Sn}_{6}$, its metallic bands depict dramatic differences. $\mathrm{C}_{6}$ consists of weakly interacting hexagonal tubular structures, and the valence and conduction bands of the $\mathbf{k} \| x y$ plane (corresponding to $\Gamma-\mathrm{M}-\mathrm{K}-\Gamma$ or $\mathrm{A}-\mathrm{L}-\mathrm{H}$ directions in $\mathrm{BZ}$ ) are flat with a wide band gap between them. However, along the $z$ direction, that is, the $\mathbf{k} \perp x y$-plane (corresponding to the $\Gamma-\mathrm{A}$ direction), energy bands are parabolic and cross the Fermi level. Under these circumstances, $\mathrm{C}_{6}$ can be viewed as if it is a bundle of conducting hexagonal rods or tubes perpendicular to the $x y$ atomic plane with a wide barrier between them that hinders the lateral conduction. Parabolic metallic bands of $\mathrm{Si}_{6}, \mathrm{Ge}_{6}$, and $\mathrm{Sn}_{6}$ derived from vertical atomic chains can also be deduced along the $\Gamma-\mathrm{A}$ direction. Additionally, bands of the $\mathbf{k} \| x y$-plane (along $\Gamma-\mathrm{M}-\mathrm{K}-\Gamma$ or $\mathrm{A}-\mathrm{L}-\mathrm{H}$ directions) also cross the Fermi level and contribute to the metallicity of these clathrate structures. Consequently, the density of states at the Fermi level, $D\left(E_{\mathrm{F}}\right)$, is high. In view of these arguments, one can expect that $\mathrm{C}_{6}$ is essentially a $1 \mathrm{D}$ conductor, but $\mathrm{Si}_{6}, \mathrm{Ge}_{6}$, and $\mathrm{Sn}_{6}$ display a $2 \mathrm{D}$ and $1 \mathrm{D}$ conduction mixed in a $3 \mathrm{D}$ crystal. We therefore expect that these directional conductivity can reflect to other measurable properties, such as thermal conductivity, etc.

Effect of Biaxial Strain. Since $\mathrm{Si}_{6}$ clathrates are open structures depicting a layered behavior, we examined the tunability of their electronic structure by biaxial strain $\epsilon$. To this end, we considered a four-layer slab of $\mathrm{Si}_{6}$ under a uniform biaxial strain and calculated the total energy $E_{\mathrm{T}}(\epsilon)$ and the interlayer spacing $c$ for a given compressive or tensile strain values. The variation of $E_{\mathrm{T}}$ with $\varepsilon$ is given in Figure 4 .

The response of $E_{\mathrm{T}}$ to the applied strain is significant; $E_{\mathrm{T}}(\epsilon)$ is symmetric with respect to the direction (sign) of small $\epsilon$, but asymmetric behavior appears for $|\epsilon|>0.03$. The variation of $c(\varepsilon)$ is not monotonic. $c$ decreases with increasing tensile strain $0.0<\epsilon<0.03$ linearly, but it increases with increasing compressive strain with a decreasing rate. Here, with the premise of modulating the superconducting transition temperature $T_{\mathcal{c}}$, we calculated the density of states at the Fermi level, $D\left(E_{\mathrm{F}}\right)$, of $\mathrm{Si}_{6}$ corresponding to its optimized structure elastically deformed under strain, $|\varepsilon|=0.0-0.05$. Densities of states (DOSs) calculated self-consistently in the energy range of $-0.5 \mathrm{eV}<E<0.5 \mathrm{eV}$ are shown in Figure $4 \mathrm{a}$,b. It is seen
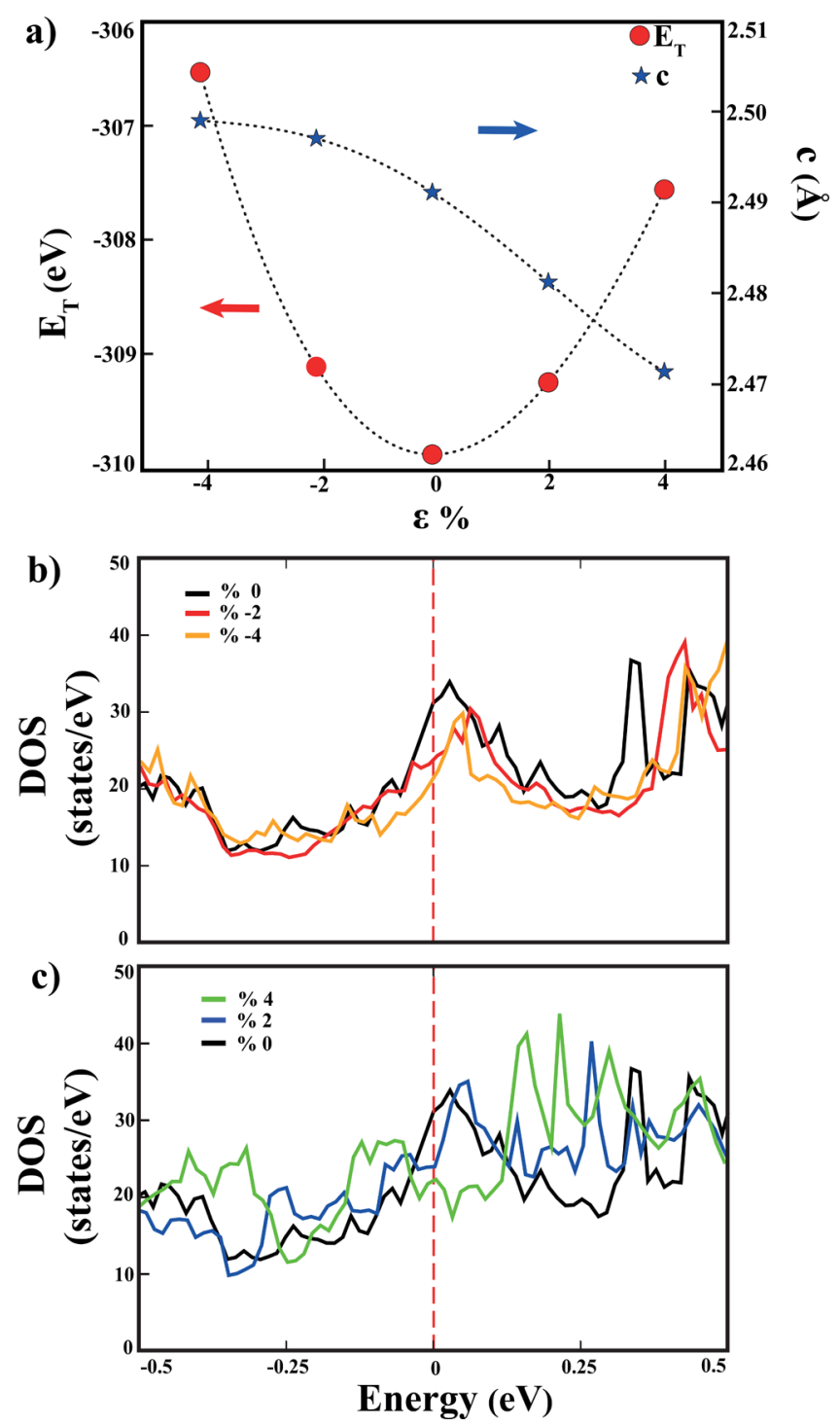

Figure 4. (a) Variation of the total energy $E_{\mathrm{T}}$ and interlayer spacing $c$ with applied biaxial strain. (b) Variation of the density of states (DOS) near the Fermi level, $D\left(E_{\mathrm{F}}\right)$, with the compressive biaxial strain. (c) Variation of the density of states near the Fermi level with the tensile biaxial strain. The zero of energy is set at the Fermi level.

that $D\left(E_{\mathrm{F}}\right)$ decreases for both increasing tensile and compressive strains. Hence, no matter what is the type of biaxial strain, let it be tensile or compressive, the transition temperature $T_{c}$, electrical conductivity $\sigma$, and thermal conductivity $\kappa$ of $\mathrm{Si}_{6}$ shall decrease with increasing $\epsilon$. This is a surprising, insofar an interesting result, and seems to be a complex interplay between the $1 \mathrm{D} / 2 \mathrm{D}$ metallicity coexisting in $\mathrm{Si}_{6}$ and reverse shifts of bands relative to the Fermi level thereof. For example, increasing 1D metallicity is compensated by decreasing $2 \mathrm{D}$ metallicity under the tensile strain.

Stability of Free-Standing Single-Layer of $\mathrm{Si}_{6}$. The atomic plane of $\mathrm{Si}_{6}$, as depicted by the top view in Figure 1 , is similar to the structure of free-standing $\omega$-borophene. ${ }^{30} \mathrm{We}$ further explored the question of whether a similar $2 \mathrm{D}$ freestanding single-layer can exist also for $\mathrm{Si}_{6}$. Notably, silicene, that is, a single layer of silicon in a buckled honeycomb structure with electrons showing massless Dirac fermion behavior and ambipolar properties, was predicted earlier to be stable. ${ }^{4,5}$ Later, silicene was synthesized on the $\operatorname{Ag}(111)$ 
(a)

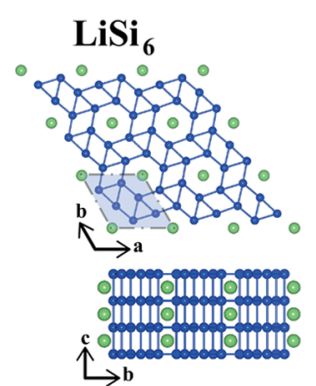

$\mathrm{NaGe}_{6}$

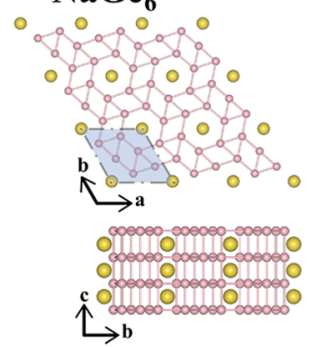

$\mathrm{KSn}_{6}$

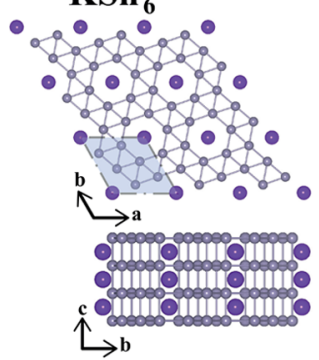

$\mathrm{MgSi}_{6}$

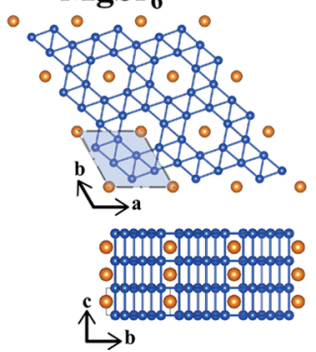

(b)
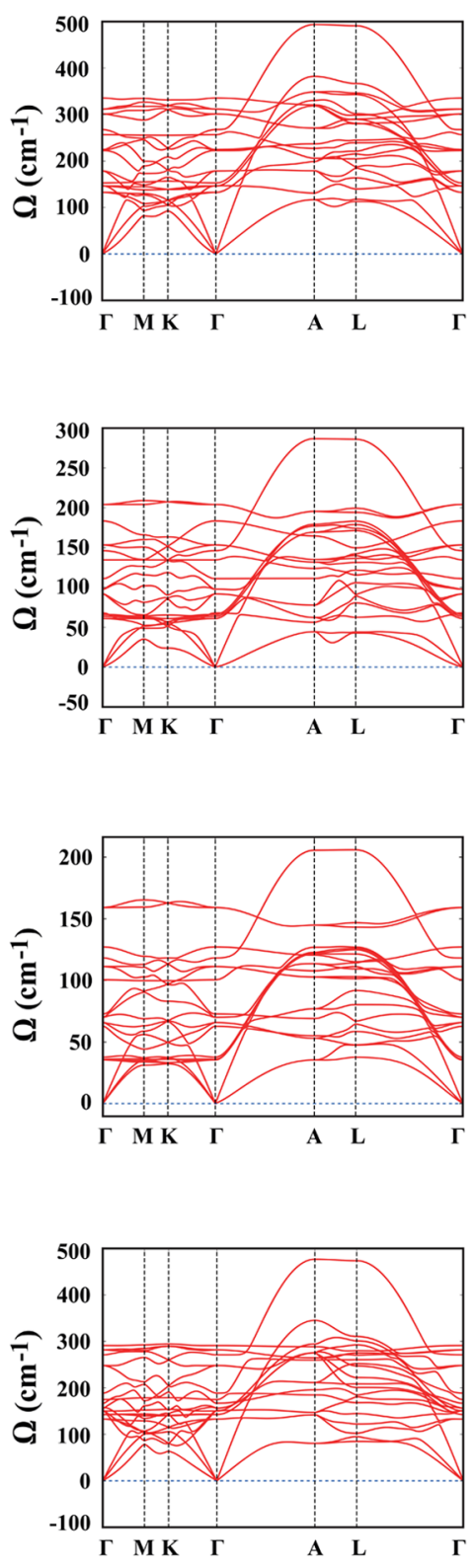

(c)
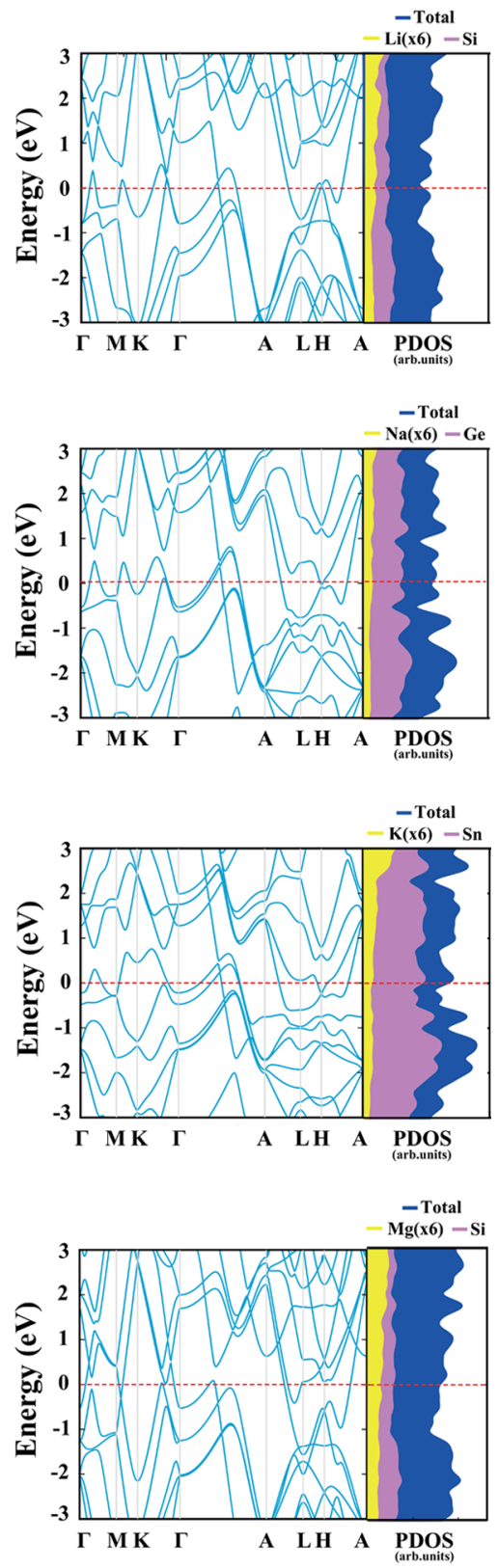

Figure 5. From left to right: (a) Top ( $x y$ plane) and side views of the optimized atomic configurations of compound clathrate structures of groupIV elements $\mathrm{XA}_{6}$ with $\mathrm{X}=\mathrm{Li}, \mathrm{Na}, \mathrm{K}, \mathrm{Mg}$ and $\mathrm{A}=\mathrm{Si}, \mathrm{Ge}, \mathrm{Sn}$. Hexagonal primitive unit cells are shaded. (b) Corresponding phonon dispersion curves, $\Omega(\mathbf{k})$, calculated for the major directions of BZ. (c) Corresponding electronic energy band structure $E_{n}(\mathbf{k})$ and the total and atom-projected densities of states. The zero of energy is set at the Fermi level and is shown by dashed lines.

substrate. ${ }^{44}$ Here, we optimized the atomic structure of a freestanding single-layer of nested hexagons. Interestingly, this planar single-layer underwent a structural transformation, whereby $\mathrm{Si}$ atoms formed a $2 \mathrm{D}$ structure consisting of deformed squares, hexagons, and octagons with 4- and 3-fold coordinated $\mathrm{Si}$ atoms having cohesion even stronger than that in silicene. Surprisingly, not only $\mathrm{Si}$, but also $\mathrm{Ge}$, compounds such as GaAs were shown to have a stable structure similar to Si. This surprising outcome of this study will be published elsewhere.

\section{D P6/M-XA 6 ALLOTROPES}

Motivated with the earlier prediction of the $\mathrm{P} 6 / \mathrm{m}-\mathrm{NaSi}_{6}$ (simply $\mathrm{NaSi}_{6}$ ) clathrate structure, ${ }^{28}$ we explored other possible compound clathrate structures $\mathrm{XA}_{6}(\mathrm{~A}=\mathrm{C}, \mathrm{Si}, \mathrm{Ge}$, and $\mathrm{Sn}$ ) and alkali, alkali earth, and $3 \mathrm{~d}$ transition metal atoms ( $\mathrm{X}=\mathrm{Li}, \mathrm{Na}, \mathrm{K}, \mathrm{Mg}, \mathrm{Ca}, \mathrm{Be}, \mathrm{Sc}, \mathrm{Ti}$ ) to acquire different properties. The stability of all these structures was analyzed by calculating the frequencies of vibration modes, as described in the previous sections. Based on this analysis, $\mathrm{XC}_{6}$ structures $(\mathrm{X}$ $=\mathrm{Li}, \mathrm{Na}$, and $\mathrm{K})$ as well as $\mathrm{XA}_{6}$ structures $(\mathrm{X}=\mathrm{Be}$ and $\mathrm{Ti} ; \mathrm{A}=$ $\mathrm{C}, \mathrm{Si}, \mathrm{Ge}, \mathrm{Sn}$ ) had imaginary frequencies in $\mathrm{BZ}$ and were found to be unstable. The remaining $16 \mathrm{XA}_{6}$ metallic structures are 
found to be stable based on the dynamical and thermal stability analyses. In Figure 5, we show optimized atomic structures, calculated phonon dispersion curves, and electronic energy band structures of the four selected clathrates. The optimized atomic structures and phonon dispersion curves of all stable $\mathrm{XA}_{6}$ structures treated in this paper are presented in the Supporting Information. The calculated values of lattice constants and minimum distances between $\mathrm{A}-\mathrm{A}$ and $\mathrm{X}-\mathrm{A}$ atoms, cohesive energy, and effective charge on $\mathrm{X}$ atoms are presented in Table 2 for the 16 stable clathrate systems.

Table 2. Values Calculated by Using PBE for Stable P6/m$\mathrm{XA}_{6}$ (or Simply $\mathrm{XA}_{6}$ ) Clathrate Structures for $\mathrm{X}=\mathrm{Li}, \mathrm{Na}, \mathrm{K}$, $\mathrm{Ca}, \mathrm{Mg}, \mathrm{Sc}$ and $\mathrm{A}=\mathrm{Si}, \mathrm{Ge}, \mathrm{Sn}^{a}$

\begin{tabular}{|c|c|c|c|c|c|c|}
\hline \multirow[b]{2}{*}{ structure } & \multicolumn{2}{|c|}{$\begin{array}{l}\text { lattice } \\
\text { constant }\end{array}$} & \multicolumn{2}{|c|}{ min. distance } & \multirow[b]{2}{*}{$\begin{array}{l}\text { cohesive energy } \\
E_{\mathrm{c}}(\mathrm{eV} / \text { atom })\end{array}$} & \multirow{2}{*}{$\begin{array}{c}\text { charge } \\
\text { transfer } \Delta \rho \\
\left(\mathrm{e}^{-}\right)\end{array}$} \\
\hline & $a(\AA)$ & $c(\AA)$ & $\begin{array}{l}d_{1} \\
(\AA)\end{array}$ & $\begin{array}{c}d_{2} \\
(\AA)\end{array}$ & & \\
\hline $\mathrm{LiSi}_{6}$ & 6.79 & 2.49 & 2.51 & 2.80 & 4.33 & -0.82 \\
\hline $\mathrm{NaSi}_{6}$ & 6.99 & 2.52 & 2.56 & 2.98 & 4.18 & -0.72 \\
\hline $\mathrm{KSi}_{6}$ & 7.34 & 2.54 & 2.53 & 3.22 & 4.32 & -0.63 \\
\hline $\mathrm{LiGe}_{6}$ & 7.31 & 2.66 & 2.60 & 2.92 & 3.70 & -0.82 \\
\hline $\mathrm{NaGe}_{6}$ & 7.42 & 2.67 & 2.79 & 3.09 & 3.65 & -0.72 \\
\hline $\mathrm{KGe}_{6}$ & 7.74 & 2.72 & 2.73 & 3.35 & 3.53 & -0.66 \\
\hline $\mathrm{LiSn}_{6}$ & 8.29 & 2.97 & 2.96 & 3.31 & 3.50 & -0.84 \\
\hline $\mathrm{NaSn}_{6}$ & 8.33 & 3.00 & 3.02 & 3.37 & 3.47 & -0.72 \\
\hline $\mathrm{KSn}_{6}$ & 8.48 & 3.06 & 3.15 & 3.25 & 3.42 & -0.66 \\
\hline $\mathrm{MgSi}_{6}$ & 6.94 & 2.56 & 2.58 & 2.93 & 4.18 & -1.44 \\
\hline $\mathrm{CaSi}_{6}$ & 7.03 & 2.64 & 2.51 & 3.04 & 4.32 & -1.13 \\
\hline $\mathrm{MgGe}_{6}$ & 7.46 & 2.72 & 2.78 & 3.10 & 3.65 & -1.24 \\
\hline $\mathrm{CaGe}_{6}$ & 7.50 & 2.80 & 2.71 & 3.21 & 3.82 & -1.16 \\
\hline $\mathrm{MgSn}_{6}$ & 8.39 & 3.01 & 3.02 & 3.37 & 3.28 & -1.07 \\
\hline $\mathrm{CaSn}_{6}$ & 8.37 & 3.09 & 3.10 & 3.47 & 3.48 & -1.17 \\
\hline $\mathrm{ScSi}_{6}$ & 6.79 & 2.63 & 2.53 & 2.89 & 4.78 & -1.09 \\
\hline
\end{tabular}

${ }^{a}$ Lattice constants in the $x y$-plane, $|\mathbf{a}|=|\mathbf{b}|=a$; along the $z$-axis, $|\mathbf{c}|=$ $c$; minimum distance between two neighboring A atoms, $d_{1}$; minimum distance between the neighboring $\mathrm{X}-\mathrm{A}$ atoms, $d_{2}$; average cohesive energy per atom, $E_{c}$; the electronic charge transfer from the $\mathrm{X}$ (metal) atom to the A (group-IV) atom, $\Delta \rho$.

It is seen in Figure $5 b$ that the inclusion of a guest alkali metal atom changes the overall vibrational spectra remarkably. A high-frequency optical mode, which is highly dispersive along the $\Gamma-\mathrm{A}-\mathrm{L}-\Gamma$ directions, is distinguished in all clathrate systems caused by an alkali metal atom. This mode is not distinguishable only in $\mathrm{NaSi}_{6}$ due to the small atomic mass difference of $\mathrm{Na}$ and $\mathrm{Si}$ atoms. Vibrational frequency range shifts to lower values as the number of row of the group-IV atom increases. Ge systems have a low-energy transverse acoustic mode along the $\Gamma-\mathrm{M}$ and $\Gamma-\mathrm{K}$ directions. Two transverse acoustic branches along the $\Gamma-\mathrm{A}$ direction are degenerate due to crystal symmetry. Nevertheless, the system under investigation does not show any vibrational anomaly, for example, phonon softening, etc.

Table 2 provides useful data for $\mathrm{XA}_{6}$ structures: Although clathrate structures $\mathrm{XA}_{6}$ in Figure 5a depict atomic structures similar to those of $A_{6}$, they have different structural parameters showing specific trends. Generally, lattice parameters $a$ and $c$ increase with increasing row number of $\mathrm{X}=\mathrm{Li}, \mathrm{Na}, \mathrm{K}$ atoms and $\mathrm{A}=\mathrm{Si}, \mathrm{Ge}, \mathrm{Sn}$ atoms. Also, for $\mathrm{X}=\mathrm{Mg}$, Ca, the lattice constants increase further. For specific structures, however, $d_{1}$ cannot follow the general trend and decreases slightly as a result of energy minimization. Generally, the average cohesive energy $E_{c}$ displays the reverse trend; namely, it decreases with the increasing row numbers of $\mathrm{X}$ and $\mathrm{A}$ atoms. Our analysis of atomic charges based on Bader $^{40}$ indicates that $\mathrm{X}$ atoms donate charge to the metallic bands derived from $\mathrm{A}$ atoms. The amount of electronic charge donated by the alkali atoms is -0.7 e and increases with the increasing row number of $\mathrm{X}$. The alkali earth atoms $\mathrm{Mg}$ and $\mathrm{Ca}$ donate a relatively more electronic charge of $-1.2 \mathrm{e}$, which decreases with the increasing row number of $\mathrm{A}$ atoms.

The overall electronic band structures of $\mathrm{XA}_{6}$ presented in Figure $5 \mathrm{c}$ and also in the Supporting Information appear to be essentially similar to those in Figure 3, except for some details. Apart from the changes in the band structures of a $\mathrm{XA}_{6}$ due to the changes in structural parameters, as well as minute change in the crystal potential of the host $A_{6}$, the major change occurs with the dipping of bands relative to the Fermi level or conversely with the raising of the Fermi level due to the electronic charge donated by $\mathrm{X}$ atoms. The downward shifts of the bands relative to the Fermi level are even more dramatic in the band structure of $\mathrm{X}=\mathrm{Mg}$ and $\mathrm{Ca}$, where more electronic charges occur to the increased population of metallic bands and, hence, to the further increase in the Fermi level.

The $\mathrm{ScSi}_{6}$ clathrate system can be stabilized at low and elevated temperatures. The optimized structure, phonon dispersion, and electronic bands of the $\mathrm{ScSi}_{6}$ system are given in Figure S7 (Supporting Information). As seen in Table $2, \mathrm{ScSi}_{6}$ has the largest cohesion among the systems considered in this work. The distinguished cohesion of $\mathrm{Sc}$ in the open hexagonal channels of $\mathrm{Si}_{6}$ can largely be attributed to the unpaired $3 \mathrm{~d}$ electron. Generally, $\mathrm{XSi}_{6}$ clathrates always have larger cohesive energies compared to $\mathrm{Ge}$ and $\mathrm{Sn}$ clathrates. As a different behavior from other $\mathrm{XA}_{6}$ clathrates, the contribution of $S c$ to electronic states at the Fermi level $D\left(E_{\mathrm{F}}\right)$ is close to the host $\mathrm{Si}$ atoms. The $\mathrm{Sc}$ atom donates less electronic charge $(1.09 \mathrm{e})$ to the metallic bands derived from $\mathrm{Si}$ compared to $\mathrm{MgSi}_{6}$ and $\mathrm{CaSi}_{6}$. Consequently, the increase in the Fermi level in the $\mathrm{ScSi}_{6}$ clathrate is not as remarkable as $\mathrm{MgSi}_{6}$ and $\mathrm{CaSi}_{6}$ yielding relatively lower $D\left(E_{\mathrm{F}}\right)$. On the other hand, it should be noted that the removal of a $\mathrm{Sc}$ atom from the $\mathrm{ScSi}_{6}$ clathrate system is rather difficult by thermal processes due to strong binding of the composite system.

\section{CONCLUSIONS}

Each group-IV element, namely, C, Si, Ge and Sn, can form a tetrahedrally coordinated cubic diamond structure in their global minimum. The fundamental band gap is as large as 5.4 $\mathrm{eV}$ for $\mathrm{C}$, but it diminishes as one goes from $\mathrm{C}$ to $\mathrm{Sn}$. In this study, we showed that $\mathrm{C}, \mathrm{Si}, \mathrm{Ge}$, and $\mathrm{Sn}$ can form also elemental clathrate structures, which are metallic with a high state density at the Fermi level. This is a rare situation since group-IV elements construct semiconducting or insulating crystals. Atomic configuration of the clathrate structure is similar for $\mathrm{Si}, \mathrm{Ge}$, and $\mathrm{Sn}$ and consists of the stacking of atomic planes of nested hexagons with an open channel at the center of the inner hexagon. While the row atoms, which are perpendicular to lateral atomic planes, form 1D metallic chains, the electrons are delocalized in the lateral atomic planes and make 2D metallic states. On the other hand, the atomic configuration of the bare clathrate structure of $\mathrm{C}$ is different and can be viewed as hexagonal tubes arranging a $2 \mathrm{D}$ hexagonal lattice. Each tube is weakly interacting with its neighbors and the crystal is insulating in the lateral plane, but it 
is $1 \mathrm{D}$ metallic along the $z$ direction. The interplay of these metallicities in different dimensionalities coexisting in one clathrate structure can give rise to interesting physical effects. By hosting metallic atoms in the open channels, $\mathrm{Si}, \mathrm{Ge}$, and $\mathrm{Sn}$ can also form stable compound clathrate structures, which are metallic. The host alkali and alkaline earth atoms donate a majority of electronic charge to metallic bands and increase the Fermi level. The open channel can also host hydrogen molecules for storage or can act as a filter for specific atoms. These new allotropes of group-IV elements with their metallic states exhibit features that can be critical fundamentally and technologically.

\section{ASSOCIATED CONTENT}

\section{S Supporting Information}

The Supporting Information is available free of charge on the ACS Publications website at DOI: 10.1021/acs.jpcc.9b03270.

Atomic structure, phonons, and electronic structures of compound clathrates of group-IV elements, $\mathrm{XA}_{6}(\mathrm{X}=\mathrm{Li}$, $\mathrm{Na}, \mathrm{K}, \mathrm{Mg}, \mathrm{Ca}, \mathrm{Sc})(\mathrm{PDF})$

\section{AUTHOR INFORMATION}

\section{Corresponding Authors}

*E-mail: ethem.akturk@adu.edu.tr. Phone: +9025621308351894. Fax: +902562135379 (E.A.).

*E-mail: ciraci@fen.bilkent.edu.tr. Phone: +903122901216. Fax: +903122664579 (S.C.).

ORCID $\odot$

Fatih Ersan: 0000-0003-0049-105X

Ethem Aktürk: 0000-0002-1615-7841

Notes

The authors declare no competing financial interest.

\section{ACKNOWLEDGMENTS}

The computational resources are provided by TÜBITAK ULAKBIM, High Performance and Grid Computing Center (TR-Grid e-Infrastructure). This research was supported by the TÜBITAK under project no. 116F059. S.C. acknowledges financial support from the Academy of Sciences of Turkey (TÜBA).

\section{REFERENCES}

(1) Harrison, W. A. Bond-Orbital Model and the Properties of Tetrahedrally Coordinated Solids. Phys. Rev. B 1973, 8, 4487.

(2) Choy, M.; Ciraci, S.; Byer, R. Bond-orbital model for secondorder susceptibilities. IEEE J. Quantum Electron. 1975, 11, 40-45.

(3) Harrison, W. A.; Ciraci, S. Bond-orbital model. II. Phys. Rev. B 1974, 10, 1516-1527.

(4) Durgun, E.; Tongay, S.; Ciraci, S. Silicon and III-V compound nanotubes: Structural and electronic properties. Phys. Rev. B 2005, 72, No. 075420.

(5) Cahangirov, S.; Topsakal, M.; Aktürk, E.; Şahin, H.; Ciraci, S. Two- and OneDimensional Honeycomb Structures of Silicon and Germanium. Phys. Rev. Lett. 2009, 102, 236804.

(6) Cahangirov, S.; Özçelik, V. O.; Xian, L.; Avila, J.; Cho, S.; Asensio, M. C.; Ciraci, S.; Rubio, A. Atomic structure of the $\sqrt{3} \times$ $\sqrt{ } 3$ phase of silicene on $\operatorname{Ag}(111)$. Phys. Rev. B 2014, 90, No. 035448. (7) Cahangirov, S.; Özçelik, V. O.; Rubio, A.; Ciraci, S. Silicite: The layered allotrope of silicon. Phys. Rev. B 2014, 90, No. 085426.

(8) Madelung, O. Semiconductors: Data Handbook; Springer Science \& Business Media, 2012.

(9) Hu, J. Z.; Spain, I. L. Phases of silicon at high pressure. Solid State Commun. 1984, 51, 263-266.
(10) Duclos, S. J.; Vohra, Y. K.; Ruoff, A. L. Experimental study of the crystal stability and equation of state of Si to 248 GPa. Phys. Rev. B 1990, 41, 12021.

(11) Minomura, S.; Drickamer, H. G. Pressure induced phase transitions in silicon, germanium and some III-V compounds. J. Phys. Chem. Solids 1962, 23, 451-456.

(12) Jamieson, J. C. Crystal structures at high pressures of metallic modifications of silicon and germanium. Science 1963, 139, 762-764.

(13) Wentorf, R. H.; Kasper, J. S. Two new forms of silicon. Science 1963, 139, 338-339.

(14) Kasper, J. S.; Richards, S. M. The crystal structures of new forms of silicon and germanium. Acta Crystallogr. 1964, 17, 752-755.

(15) Vohra, Y. K.; Brister, K. E.; Desgreniers, S.; Ruoff, A. L.; Chang, K. J.; Cohen, M. L. Phase-Transition Studies of Germanium to 1.25 Mbar. Phys. Rev. Lett. 1986, 56, 1944-1947.

(16) Mujica, A.; Rubio, A.; Muñoz, A.; Needs, R. J. High-pressure phases of group-IV, III-V, and II-VI compounds. Rev. Mod. Phys. 2003, 75, 863.

(17) Crain, J.; Ackland, G. J.; Maclean, J. R.; Piltz, R. O.; Hatton, P. D.; Pawley, G. S. Reversible pressure-induced structural transitions between metastable phases of silicon. Phys. Rev. B 1994, 50, 13043.

(18) Malone, B. D.; Sau, J. D.; Cohen, M. L. Ab initio survey of the electronic structure of tetrahedrally bonded phases of silicon. Phys. Rev. B 2008, 78, No. 035210.

(19) Zhao, Y.-X.; Buehler, F.; Sites, J. R.; Spain, I. L. New metastable phases of silicon. Solid State Commun. 1986, 59, 679-682.

(20) Rapp, L.; Haberl, B.; Pickard, C. J.; Bradby, J. E.; Gamaly, E. G.; Williams, J. S.; Rode, A. V. Experimental evidence of new tetragonal polymorphs of silicon formed through ultrafast laser-induced confined microexplosion. Nat. Commun. 2015, 6, 7555.

(21) McMahon, M. I.; Nelmes, R. J.; Wright, N. G.; Allan, D. R. Pressure dependence of the Imma phase of silicon. Phys. Rev. B 1994, $50,739$.

(22) Hanfland, M.; Schwarz, U.; Syassen, K.; Takemura, K. Crystal structure of the highpressure phase silicon VI. Phys. Rev. Lett. 1999, $82,1197$.

(23) Mylvaganam, K.; Zhang, L. C.; Eyben, P.; Mody, J.; Vandervorst, W. Evolution of metastable phases in silicon during nanoindentation: mechanism analysis and experimental verification. Nanotechnology 2009, 20, 305705.

(24) Gryko, J.; McMillan, P. F.; Marzke, R. F.; Ramachandran, G. K.; Patton, D.; Deb, S. K.; Sankey, O. F. Low-density framework form of crystalline silicon with a wide optical band gap. Phys. Rev. B 2000, 62, R7707.

(25) Stefanoski, S.; Malliakas, C. D.; Kanatzidis, M. G.; Nolas, G. S. Synthesis and Structural Characterization of $\mathrm{Na}_{\mathrm{x}} \mathrm{Si}_{136}(0<\mathrm{x} \leq 24)$ Single Crystals and Low-Temperature Transport of Polycrystalline Specimens. Inorg. Chem. 2012, 51, 8686-8692.

(26) Kurakevych, O. O.; Strobel, T. A.; Kim, D. Y.; Muramatsu, T.; Struzhkin, V. V. Na-Si clathrates are high-pressure phases: A meltbased route to control stoichiometry and properties. Cryst. Growth Des. 2013, 13, 303-307.

(27) Kim, D. Y.; Stefanoski, S.; Kurakevych, O. O.; Strobel, T. A. Synthesis of an openframework allotrope of silicon. Nat. Mater. 2015, 14, 169.

(28) Sung, H.-J.; Han, W. H.; Lee, I.-H.; Chang, K. J. Superconducting Open-Framework Allotrope of Silicon at Ambient Pressure. Phys. Rev. Lett. 2018, 120, 157001.

(29) Lee, J.; Lee, I.-H.; Lee, J. Unbiased Global Optimization of Lennard-Jones Clusters for $\mathrm{N} \leq 201$ Using the Conformational Space Annealing Method. Phys. Rev. Lett. 2003, 91, No. 080201.

(30) Liu, D.; Tománek, D. Effect of Net Charge on the Relative Stability of 2D Boron Allotropes. Nano Lett. 2019, 19, 1359-1365.

(31) Blöchl, P. E. Projector augmented-wave method. Phys. Rev. B 1994, 50, 17953.

(32) Kresse, G.; Furthmüller, J. Efficient iterative schemes for ab initio total-energy calculations using a plane-wave basis set. Phys. Rev. B 1996, 54, 11169. 
(33) Kresse, G.; Furthmüller, J. Efficiency of ab-initio total energy calculations for metals and semiconductors using a plane-wave basis set. Comput. Mater. Sci. 1996, 6, 15-50.

(34) Perdew, J. P.; Burke, K.; Ernzerhof, M. Generalized Gradient Approximation Made Simple. Phys. Rev. Lett. 1996, 77, 3865.

(35) Grimme, S. Semiempirical GGA-type density functional constructed with a long-range dispersion correction. J. Comput. Chem. 2006, 27, 1787-1799.

(36) Broyden, C. G. The convergence of a class of double-rank minimization algorithms 1. general considerations. IMA J. Appl. Math. 1970, 6, 76-90.

(37) Broyden, C. G. The convergence of a class of double-rank minimization algorithms: 2. The new algorithm. IMA J. Appl. Math. 1970, 6, 222-231.

(38) Methfessel, M.; Paxton, A. T. High-precision sampling for Brillouin-zone integration in metals. Phys. Rev. B 1989, 40, 3616.

(39) Monkhorst, H. J.; Pack, J. D. Phys. Rev. B 1976, 13, 5188.

(40) Henkelman, G.; Arnaldsson, A.; Jónsson, H. A fast and robust algorithm for Bader decomposition of charge density. Comput. Mater. Sci. 2006, 36, 354-360.

(41) Baroni, S.; de Gironcoli, S.; Dal Corso, A.; Giannozzi, P. Phonons and related crystal properties from density-functional perturbation theory. Rev. Mod. Phys. 2001, 73, 515-562.

(42) Togo, A.; Tanaka, I. First principles phonon calculations in materials science. Scr. Mater. 2015, 108, 1-5.

(43) Momma, K.; Izumi, F. VESTA 3 for three-dimensional visualization of crystal, volumetric and morphology data. J. Appl. Crystallogr. 2011, 44, 1272-1276.

(44) Vogt, P.; De Padova, P.; Quaresima, C.; Avila, J.; Frantzeskakis, E.; Asensio, M. C.; Resta, A.; Ealet, B.; Le Lay, G. Silicene: Compelling Experimental Evidence for Graphenelike Two-Dimensional Silicon. Phys. Rev. Lett. 2012, 108, 155501. 